International Journal of Distributed and Parallel Systems (IJDPS) Vol.3, No.3, May 2012

\title{
Performance Evaluation of an OFDM SYSTEM UNDER THE INFLUENCE OF PHASE NoISE AND CARRIER FREQUENCY OFFSET
}

\author{
${\text { Gurpreet } \text { Kaur }^{1} \text { and Partha Pratim Bhattacharya }}^{2}$ \\ Department of Electronics and Communication Engineering \\ Faculty of Engineering and Technology \\ Mody Institute of Technology \& Science (Deemed University) \\ Lakshmangarh, Dist. Sikar, Rajasthan, \\ Pin - 332311, India \\ ${ }^{1}$ gur1487preetegmail.com \\ ${ }^{2}$ hereispartha@gmail.com
}

\begin{abstract}
The two most important parameters to be considered while designing an OFDM based communication system are phase noise and carrier frequency offset (CFO). It has been observed that the OFDM systems are very sensitive to phase noise and CFO, and an accurate prediction of the tolerable values of these parameters can help the system to relax the specifications. This paper analyses the performance of OFDM systems in the presence of phase noise and carrier frequency offset. Results clearly show the SINR penalty in the OFDM system due to phase noise and CFO.
\end{abstract}

\section{KEYWORDS}

OFDM Wireless communication system, Phase noise, carrier frequency offset (CFO), signal to interference plus noise ratio (SINR), Rayleigh fading.

\section{INTRODUCTION TO ORTHOgONAL FrEQUENCY DIVISION MultipleXing (OFDM)}

With the growing demand of high speed internet, extensive research has been carried out to develop systems which can combat multipath effects. OFDM (Orthogonal Frequency Division Multiplexing) has been developed to combat this effect and make better use of the system. It has been adopted for Digital Video Terrestrial Broadcasting (DVB) and for the European Digital Audio Broadcasting (DAB) standards.

Phase noise is an important parameter that should be carefully considered while dealing with any of the above communication systems because an accurate prediction of the tolerable phase noise can allow the system to relax specifications. This is an important point to be considered in OFDM communication systems with frequencies above $25 \mathrm{GHz}$, as suggested in some European ACTS projects dealing with LMDS (Local-Multipoint Distribution Systems) [1]. The effect of phase noise in OFDM and the degradation caused by it have been analyzed by several 
authors [2]-[5].

Carrier frequency offset (CFO) exist between user terminals and the base station because of the Doppler effect and precision limitation of oscillators. OFDM systems are very sensitive to CFO [6], which leads to performance degradation by introducing inter-carrier-interference (ICI) [7]. The purpose of this paper is to analyze the performance of an OFDM wireless communication system in the combined effect of phase noise and carrier frequency offset in Rayleigh fading environment.

One of the best ways to mitigate the effect of multipath is to use orthogonal frequency division multiplexing communication systems. The delay spread is of some microseconds for the reception purposes in built up regions. The channel is highly frequency selective and sophisticated equalization techniques are required to achieve high bit rate transmissions. However, a combination of OFDM and coding associated with interleaving in the frequency domain (COFDM) can take advantage from the diversity associated to multipath [8].

In an OFDM system the spectrum associated to each elemental data is a small portion of the total bandwidth (B), which is divided in N sub-channels. Each of them is modulated with one information symbol and they are all multiplexed in frequency. If $\mathrm{T}$ represents the OFDM symbol duration, that is: $\mathrm{T}=\mathrm{N} / \mathrm{B}$, the $\mathrm{N}$ sub-carriers are placed in the frequencies:

$$
f_{k}=f_{0}+\frac{k}{T}, \quad k=0,1 \ldots N-1
$$

The different sub-channels are allowed to overlap so as to increase the bandwidth efficiency.

Unfortunately OFDM is very much sensitive to the synchronization errors such as Carrier Frequency Offset (CFO) and phase noise or timing jitter.

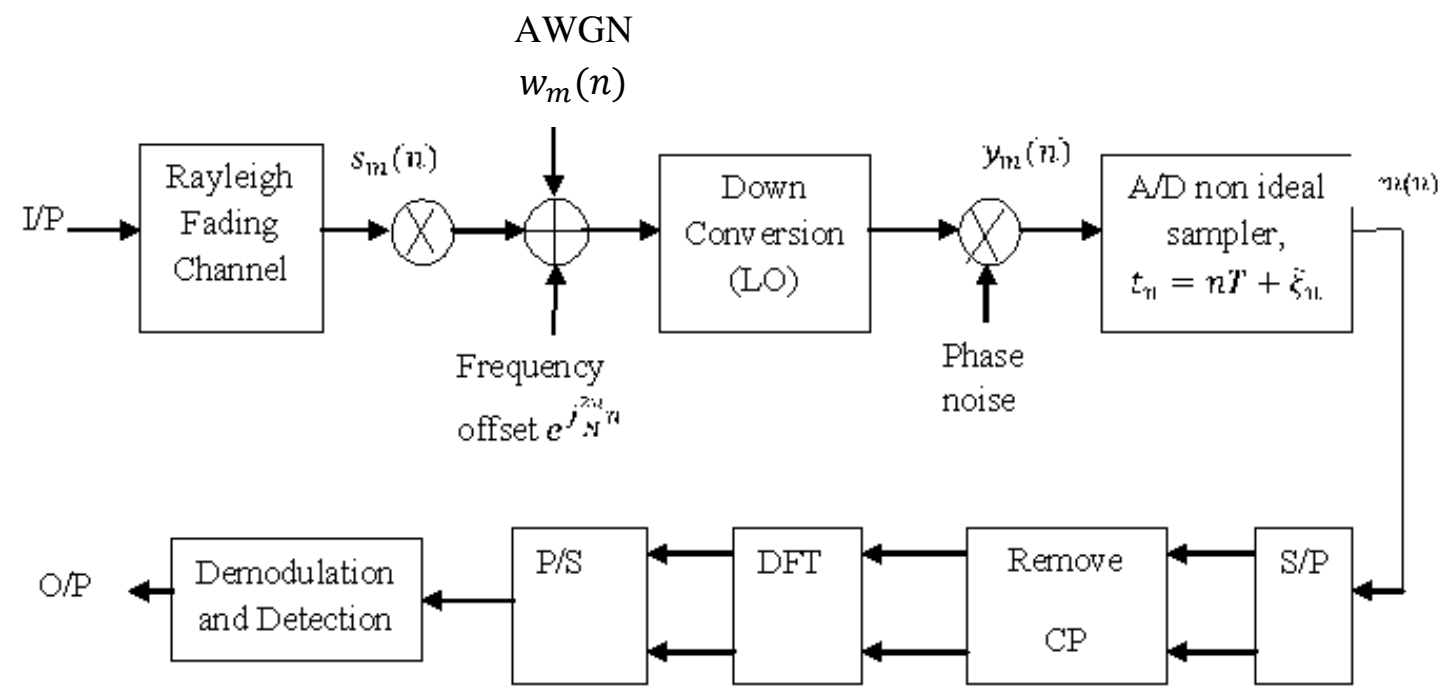

Fig. 1 OFDM system model (receiver) in the presence of CFO, phase noise and timing jitter over Rayleigh fading channel. 
The OFDM system model is shown in Fig. 1[9]. Here, the input is considered to be passed through a Rayleigh fading environment and then additive white Gaussian noise is added to it. After the addition of AWGN, phase noise is incorporated to the system and there after timing jitter. The signal is then passed through serial to parallel converter; and cyclic prefix is removed after this. Finally the signal undergoes Discrete Fourier Transformation, parallel to serial conversion and a demodulation process. Following equation gives the $\mathrm{N}$ point complex modulation sequence transmitted by OFDM signal for the $m^{\text {th }}$ symbol:

$$
x_{m}(n)=\sum_{k=0}^{N-1} X_{m}(k) e^{j \frac{2 \pi}{N} n k}
$$

where $\mathrm{n}$ ranges from 0 to $N+N_{g}-1$.

After passing this signal through a Rayleigh fading channel and then through the local oscillator, the received signal impaired by AWGN and phase noise can be modeled as:

$$
y_{m}(n)=\left[\sum_{k=0}^{N-1} X_{m}(k) H_{m}(k) e^{j \frac{2 \pi}{N} n(k+)}\right] e^{j \Phi_{m}(n)}+w_{m}(n)
$$

or,

$$
y_{m}(n)=s_{m}(n) e^{j \Phi_{m}(n)}+w_{m}(n)
$$

where,

$$
s_{m}(n)=\sum_{k=0}^{N-1} X_{m}(k) H_{m}(k) e^{j \frac{2 \pi}{N} n(k+)}
$$

In (3) $H_{m}(k)$ is the transfer function of the Rayleigh fading channel at the frequency of the $k^{t h}$ carrier and $w_{m}(n)$ is the complex envelope of AWGN with zero mean and variance $\sigma^{2}$.

Assuming $\Phi_{m}(n)$ is small so that:

Substituting (5) into (4) we get:

$$
e^{j \Phi_{m}(n)}=1+j \Phi_{m}(n)
$$

$$
y_{m}(n)=s_{m}(n)+s_{m}(n) j \Phi_{m}(n)+w_{m}(n)
$$

After DFT and by dropping the subscript ' $m$ ' (6) yields

$$
Y(k)=S(k)+S(k) \otimes j \Theta(k)+W(k)
$$

where $\mathrm{S}(\mathrm{k}), \Theta(\mathrm{k})$ and $\mathrm{W}(\mathrm{k})$ are the DFT responses of $s_{m}(n), \Phi_{m}(n)$ and $w_{m}(n)$ respectively and $\otimes$ denotes the circular convolution operation.

\section{Phase Noise}

Phase noise effects which are introduced by the local oscillator in any receiver can only be 
removed by improving the performance of the oscillator itself. Therefore the determination of how much phase noise a receiver can withstand while maintaining the required performance is of utmost importance. Phase noise can generally be seen as a parasitic phase modulation in the oscillator's signal, which ideally would be a unique carrier with constant amplitude and frequency. For simulation purposes it has been modeled as a phase modulation of the carrier.

\subsection{Phase Noise in OFDM}

A theoretical analysis of phase noise effects in OFDM signals is carried out by many authors. The complex envelope of the transmitted OFDM signal for a given OFDM symbol, sampled with sampling frequency $f_{s}=B$, is:

$$
x(n)=\sum_{k=0}^{N-1} s_{k} \cdot e^{j \frac{2 \pi}{N} k n}
$$

where $\mathrm{n}=0,1, \ldots, \mathrm{N}-1$.

This symbol is actually extended with a Time Guard in order to cope with multipath delay spread. We will not consider this prefix since it is eliminated in the receiver for the sake of simplicity. Assuming that the channel is flat, the signal is only affected by phase noise at the receiver:

$$
r(n)=x(n) \cdot e^{j \Phi(n)}
$$

The received signal is a Orthogonal Frequency Division De multiplexed (OFDD) signal by means of a Discrete Fourier Transform. For the purpose of separating the signal and noise terms, let us suppose that $\Phi(\mathrm{n})$ is small, so that:

$$
e^{j \Phi(n)} \approx 1+j \Phi(n)
$$

In this case, the de multiplexed signal is:

$$
\begin{gathered}
y(k) \approx s_{k}+\frac{j}{N} \sum_{r=0}^{N-1} s_{r} \sum_{n=0}^{N-1} \Phi(n) \cdot e^{j\left(\frac{2 \pi}{N}\right)(r-k) n} \\
y(k) \approx s_{k}+e_{k}
\end{gathered}
$$

Thus we have an error term $e_{k}$ for each sub-carrier and which results from some combination of all of them and is added to the useful signal. The signal to noise ratio degradation $\left(\operatorname{degr}_{S N R}\right)$ caused by phase noise is the same in OFDM and signal carrier systems, given that phase noise variance is small $\left(\sigma^{2} \ll 1\right)$ it follows the expression [10] :

$$
\operatorname{degr}_{S N R}=10 \cdot \log \left(1+\sigma^{2} \frac{E_{S}}{N_{0}}\right) d B .
$$

In this equation $E_{S}$ represents the symbol energy and $N_{0}$ is the power spectral density of additive white Gaussian noise. The most common way to characterize oscillator's phase noise is the single-side-band phase noise power density function $L\left(f_{m}\right)$, which represents the ratio (in $\mathrm{dBc}$; 'c' stands for carrier) between the single-side-band noise power in a $1 \mathrm{~Hz}$ bandwidth at a distance $f_{m}$ from the carrier and the carrier power [11].

This characterization is normally performed by using a spectrum analyzer which provides the power spectral density of the equipment's phase noise $\left(N_{0 p}\right)$ in relation to the carrier power 
(C). From these measurements, given that the phase noise has a zero mean as we have assumed and it extends up to a frequency $b$ (either because phase noise is band-limited or due to the presence of filtering in the receiver), its variance can be found as [11]:

$$
\sigma^{2}=\int_{0}^{b}\left(\frac{2 N_{0 p}}{C}\right) d f
$$

\section{CARrier Frequency OffSet (CFO) in OFDM}

The absolute value of the actual CFO $f$, is either an integer multiple or a fraction of $\Delta f$, or the sum of them. If $f$ is normalized to the subcarrier spacing $\Delta f$, then the resulting normalized CFO of the channel can be generally expressed as:

$$
=\frac{f}{\Delta f}=\delta+\epsilon
$$

where $\delta$ is an integer and $|\epsilon| \leq 0.5$. The influence of an integer CFO on OFDM system[12] is different from the influence of a fractional CFO. In the event that $\delta \neq 0$ and $\varepsilon=0$, symbols transmitted on a certain subcarrier, e.g., subcarrier $\mathrm{k}$, will shift to another subcarrier $k_{\delta}, k_{\delta}=$ $k+\delta \bmod N-1$. As the ICI effect is focussed, normalized CFO is considered,

$$
=\frac{f}{\Delta f}=\epsilon
$$

Since no ICI is caused by an integer CFO, relative CFO $(\varepsilon)$ to assumed to be a Gaussian process, statistically independent of the input signal, with zero mean and variance $\sigma^{2}$.

\section{Formulation FOR SINR AND Simulation PARAMETERS}

In the presence of CFO, phase noise, timing jitter and Rayleigh fading the OFDM system performance can be given by the following SINR expression [13]:

$$
\begin{array}{r}
\operatorname{SINR}\left(, \sigma_{u}^{2}, \xi, \alpha\right) \geq \frac{\gamma_{i n} \alpha^{2}(1-\xi)\left\{\operatorname{sinc}^{2}(\pi)\right\}}{1+\gamma_{i n} \alpha^{2}(1-\xi)\left[0.5947(\sin \pi)^{2}+\left\{\frac{\sigma_{u}^{2}}{2 N} \operatorname{sinc}^{2}(\pi) \sum_{r=1}^{N-1} \frac{1}{\sin ^{2}\left(\frac{\pi r}{N}\right)}\right\}\right]+\gamma_{i n} \alpha^{2} \xi} \\
\quad ;|| \leq 0.5,|\xi| \leq 1 . \quad(17)
\end{array}
$$

where $?$ is the normalized CFO, $\sigma_{u}^{2}$ is the variance of phase noise, $\xi$ denotes timing jitter and $\alpha$ corresponds to the channel attenuation/gain parameter in Rayleigh fading environment. $\gamma_{\text {in }}$ is the input SNR, $\mathrm{N}$ is the number of sub-carriers in the channel. Therefore the SINR expression in the presence of phase noise and CFO without timing jitter and considering a non-fading environment $(\alpha=1)$ can be expressed as: 
International Journal of Distributed and Parallel Systems (IJDPS) Vol.3, No.3, May 2012

$$
\begin{gathered}
\operatorname{SINR}\left(, \sigma_{u}^{2}\right) \geq \frac{\gamma_{i n}\left\{\operatorname{sinc}^{2}(\pi)\right\}}{1+\gamma_{i n}\left[0.5947(\sin \pi)^{2}+\left\{\frac{\sigma_{u}^{2}}{2 N} \operatorname{sinc}^{2}(\pi) \sum_{r=1}^{N-1} \frac{1}{\sin ^{2}\left(\frac{\pi r}{N}\right)}\right\}\right]} \\
; \| \leq 0.5
\end{gathered}
$$

It can be seen that in the presence of CFO and phase noise the SINR expression in (18) is a function of these two parameters as well as other critical system parameters. Table 1 shows the system and channel parameters considered for the simulation.

Table 1. System and channel parameters for simulation

\begin{tabular}{|c|c|}
\hline Number of sub carriers $(\mathrm{N})$ & 64 \\
\hline Channel type & Rayleigh fading channel \\
\hline Input SNR values & $10,15,20,25 \mathrm{~dB}$. \\
\hline Channel attenuation/gain parameter & Ideal $(1)$ \\
\hline
\end{tabular}

\section{RESULTS AND DISCUSSION}

Simulation has been carried out using MATLAB. Figure 2, 3 and 4 show the plot of SINR versus normalized CFO for various values of variance of phase noise $\left(\sigma_{u}^{2}\right)$. Results are plotted for different values of input SNR $\left(\gamma_{i}\right)$ of $10 \mathrm{~dB}, 15 \mathrm{~dB}, 20 \mathrm{~dB}$ and $25 \mathrm{~dB}$. It can be seen from Figures 2, 3 and 4 that as the variance of phase noise increases the value of SINR decreases. In the Figure 2 for an input SNR of $10 \mathrm{~dB}$ and variance of phase noise of 0.015 , the initial SINR is $10 \mathrm{~dB}$ and then gradually decreases. The observations are similar for all SNR values. Similarly, Figure 3 shows SINR vs. normalized CFO for an input SNR of $10 \mathrm{~dB}$ and variance of phase noise of 0.33 , the SINR is initially $-1 \mathrm{~dB}$ and decreases gradually. Figure 4 shows that for variance of phase noise of 1.33 and input SNR being $10 \mathrm{~dB}$ the SINR starts at $-12.8 \mathrm{~dB}$ and then decreases as normalized CFO increases. The nature of the variations are same for all input SNR values. But the SINR value decreases when the variance of phase noise increases. 
International Journal of Distributed and Parallel Systems (IJDPS) Vol.3, No.3, May 2012

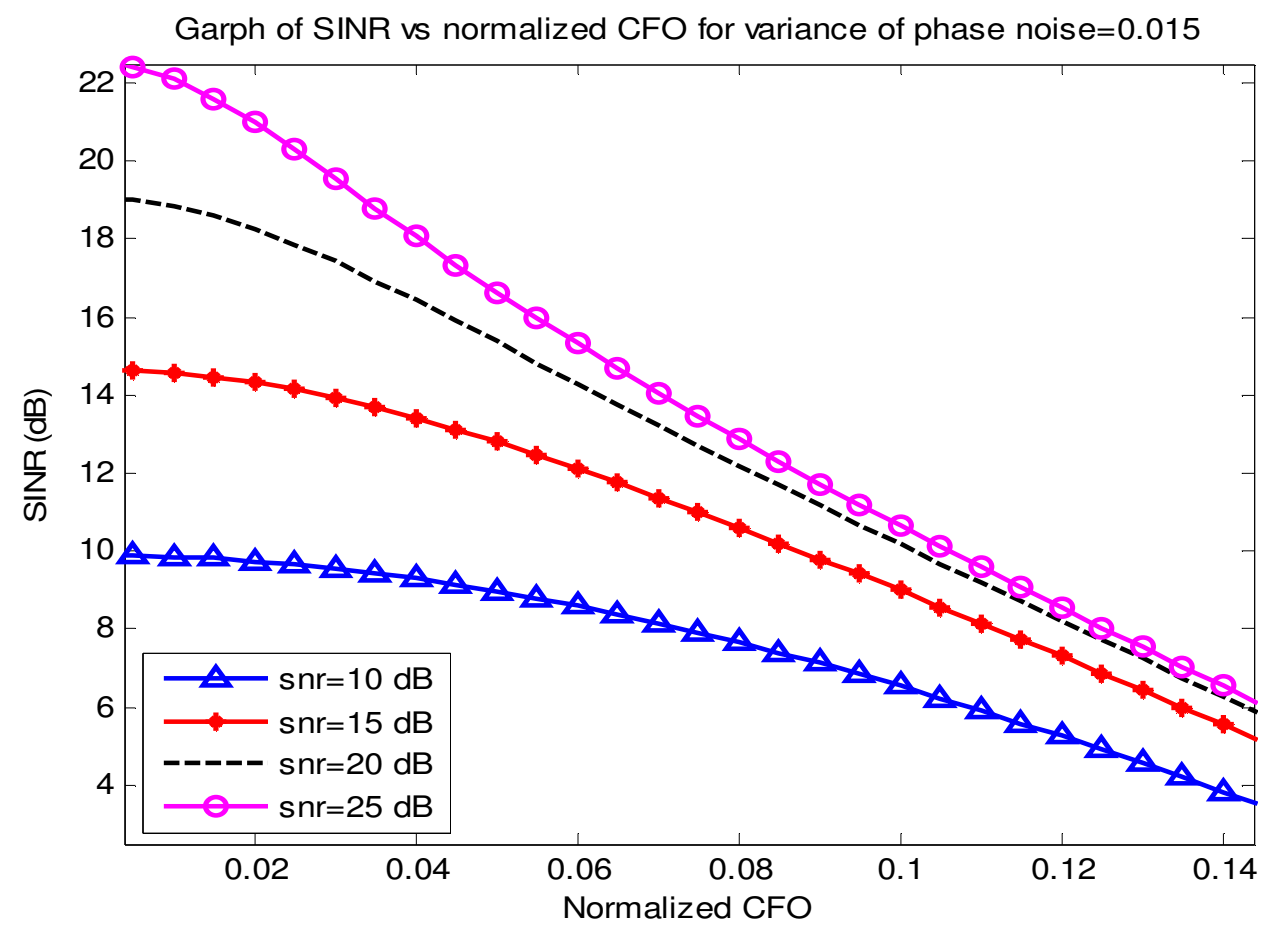

Fig. 2 SINR versus normalized CFO (variance of phase noise=0.015)

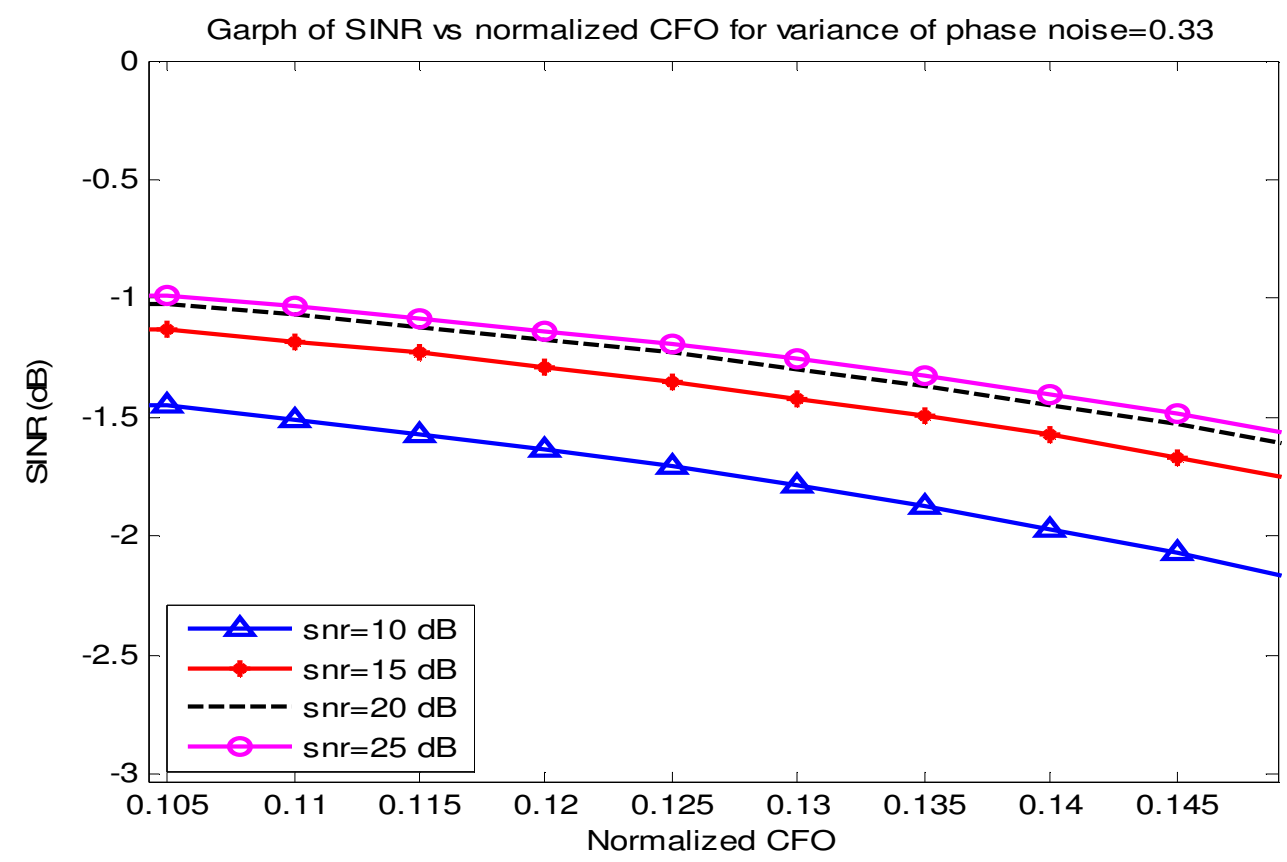

Fig. 3 SINR versus normalized CFO (variance of phase noise $=0.33$ ) 
International Journal of Distributed and Parallel Systems (IJDPS) Vol.3, No.3, May 2012

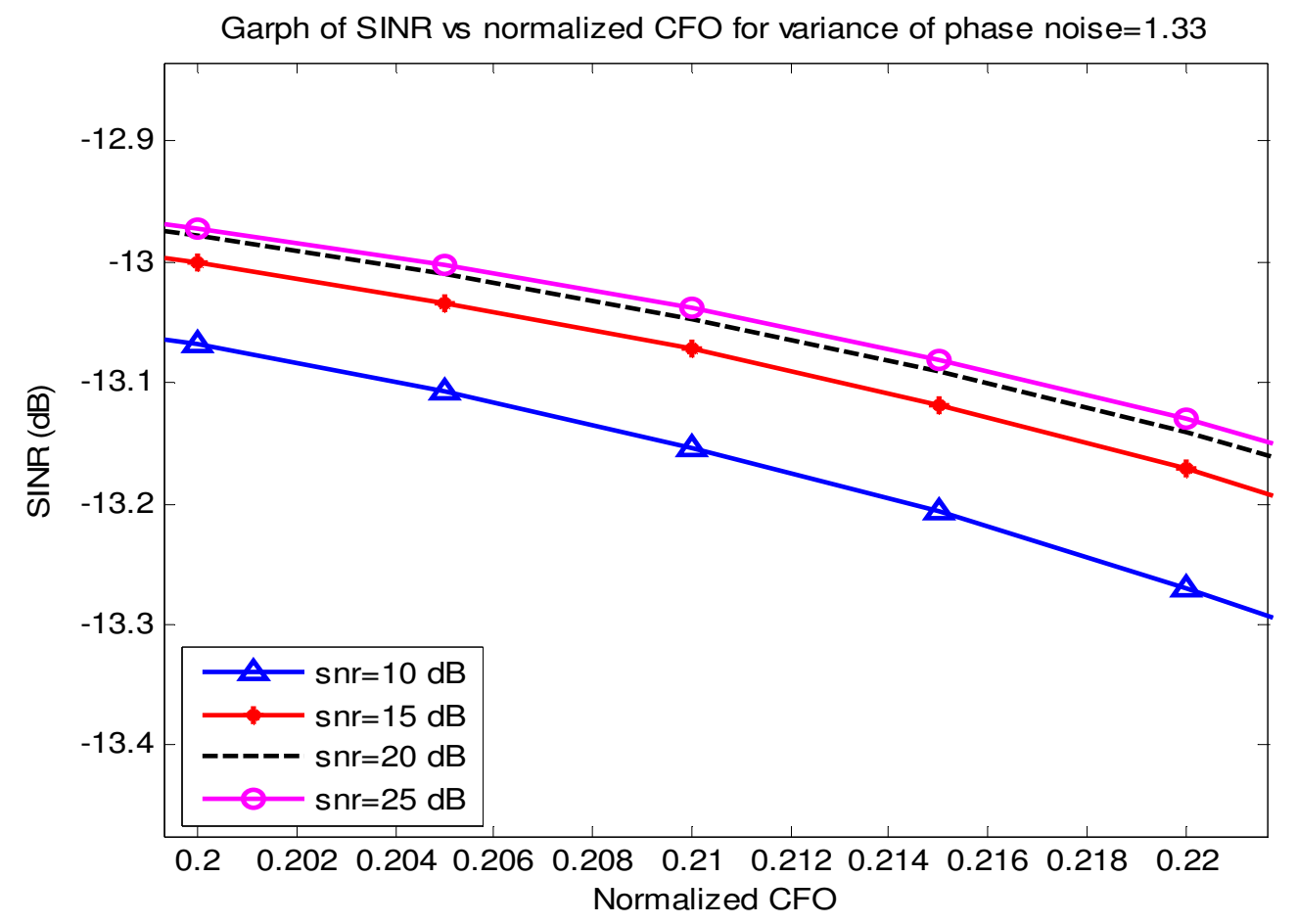

Fig. 4 SINR versus normalized CFO (variance of phase noise $=1.33$ )

Figures 5, 6, 7, 8, 9 and 10 show the variation of SINR with variance of phase noise $\left(\sigma_{u}^{2}\right)$ for various values of normalized CFO of $0.05,0.1,0.15,0.20,0.25$ and 0.30 . The results are plotted for values of input SNR $\left(\gamma_{i}\right)$ of $10 \mathrm{~dB}, 15 \mathrm{~dB}, 20 \mathrm{~dB}$ and $25 \mathrm{~dB}$. From Figures 5, 6, 7, 8, 9 and 10 it can be seen that as the value of normalized CFO increases the SINR value decreases. For an input SNR of $10 \mathrm{~dB}$ and normalized CFO of 0.05, 0.1, 0.15, 0.2, 0.25 and 0.3, the initial values of SINR are 9, 6.5, 3, -1.5, -7.9, -21.25 dB respectively, which shows a gradual decrement. 
International Journal of Distributed and Parallel Systems (IJDPS) Vol.3, No.3, May 2012

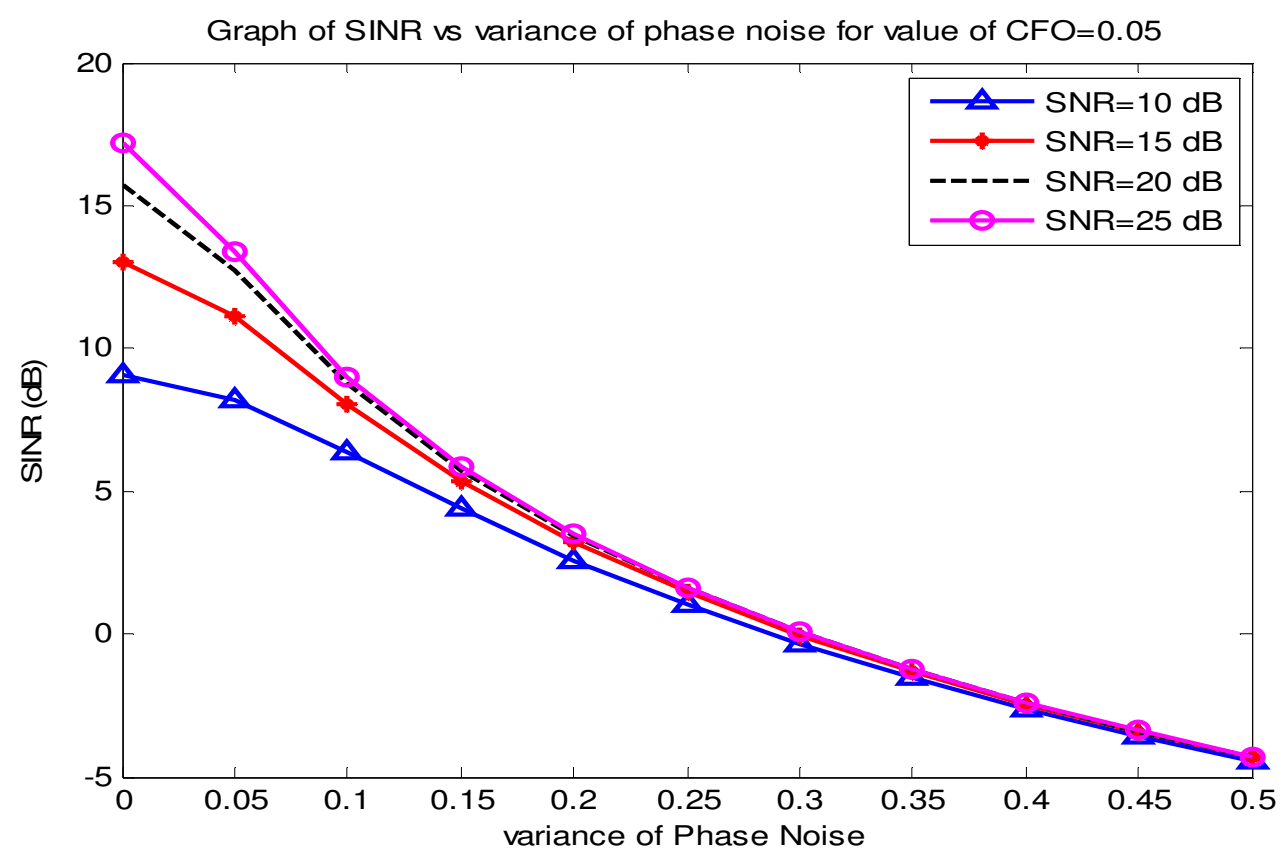

Fig. 5 SINR versus variance of phase noise (normalized $\mathrm{CFO}=0.05$ )

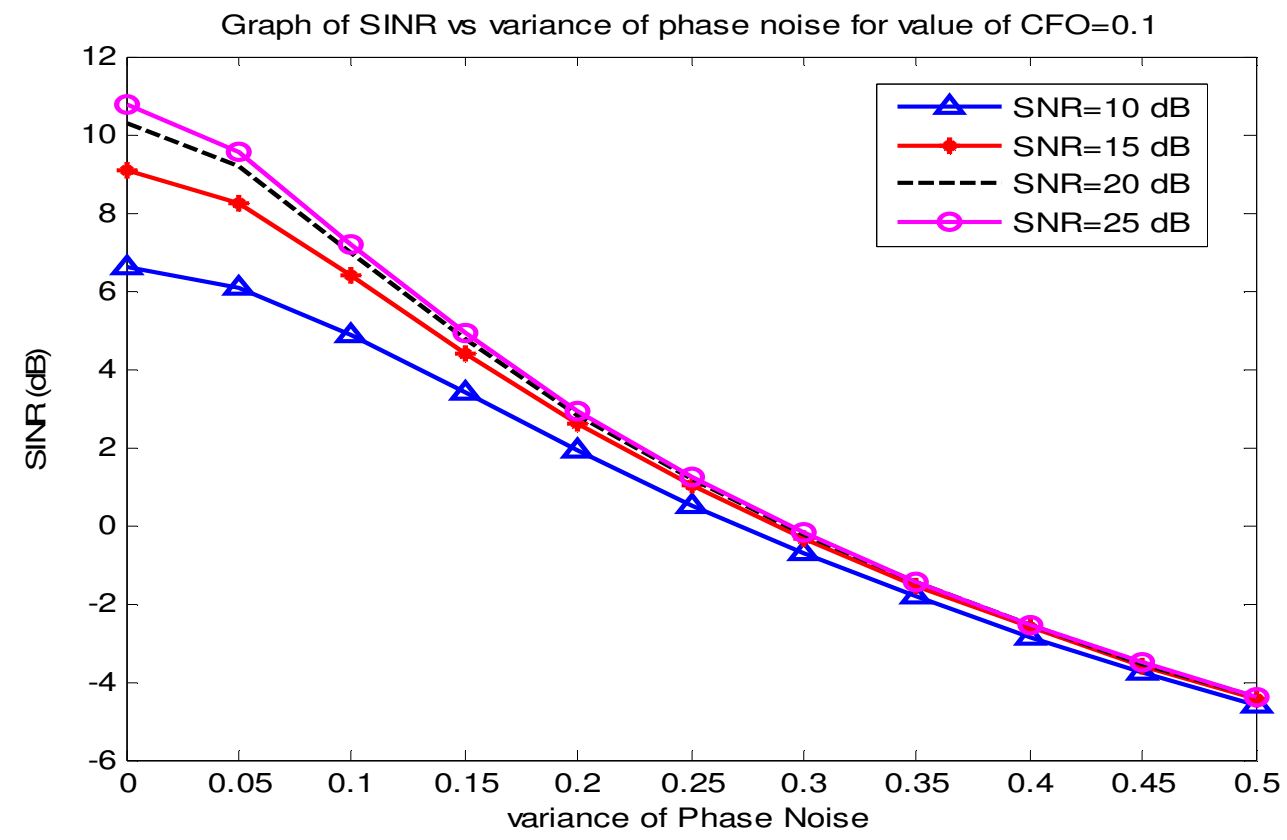

Fig. 6 SINR versus variance of phase noise (normalized $\mathrm{CFO}=0.1$ ) 
International Journal of Distributed and Parallel Systems (IJDPS) Vol.3, No.3, May 2012

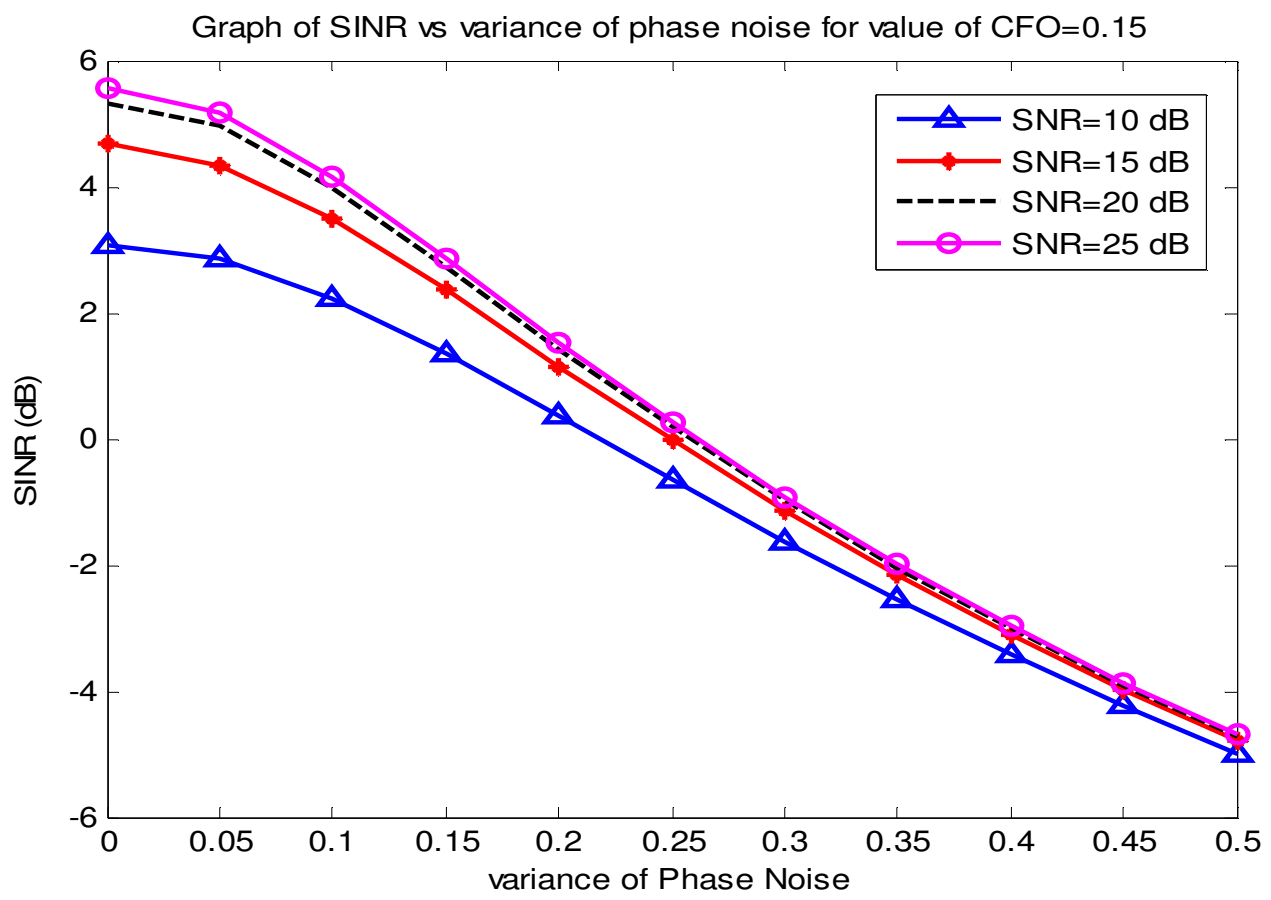

Fig. 7 SINR versus variance of phase noise (normalized $\mathrm{CFO}=0.15$ )

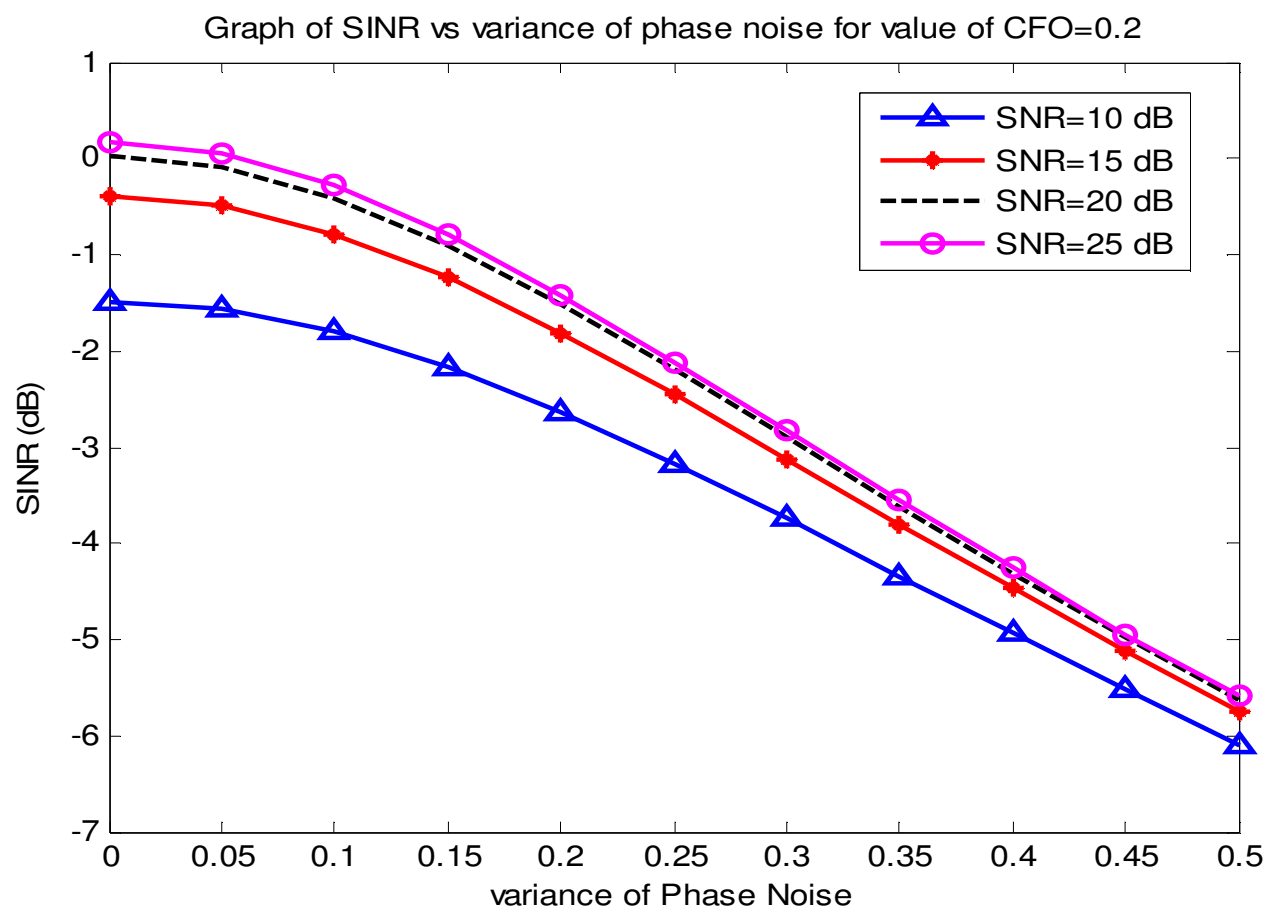

Fig. 8 SINR versus variance of phase noise (normalized $\mathrm{CFO}=0.2$ ) 
International Journal of Distributed and Parallel Systems (IJDPS) Vol.3, No.3, May 2012

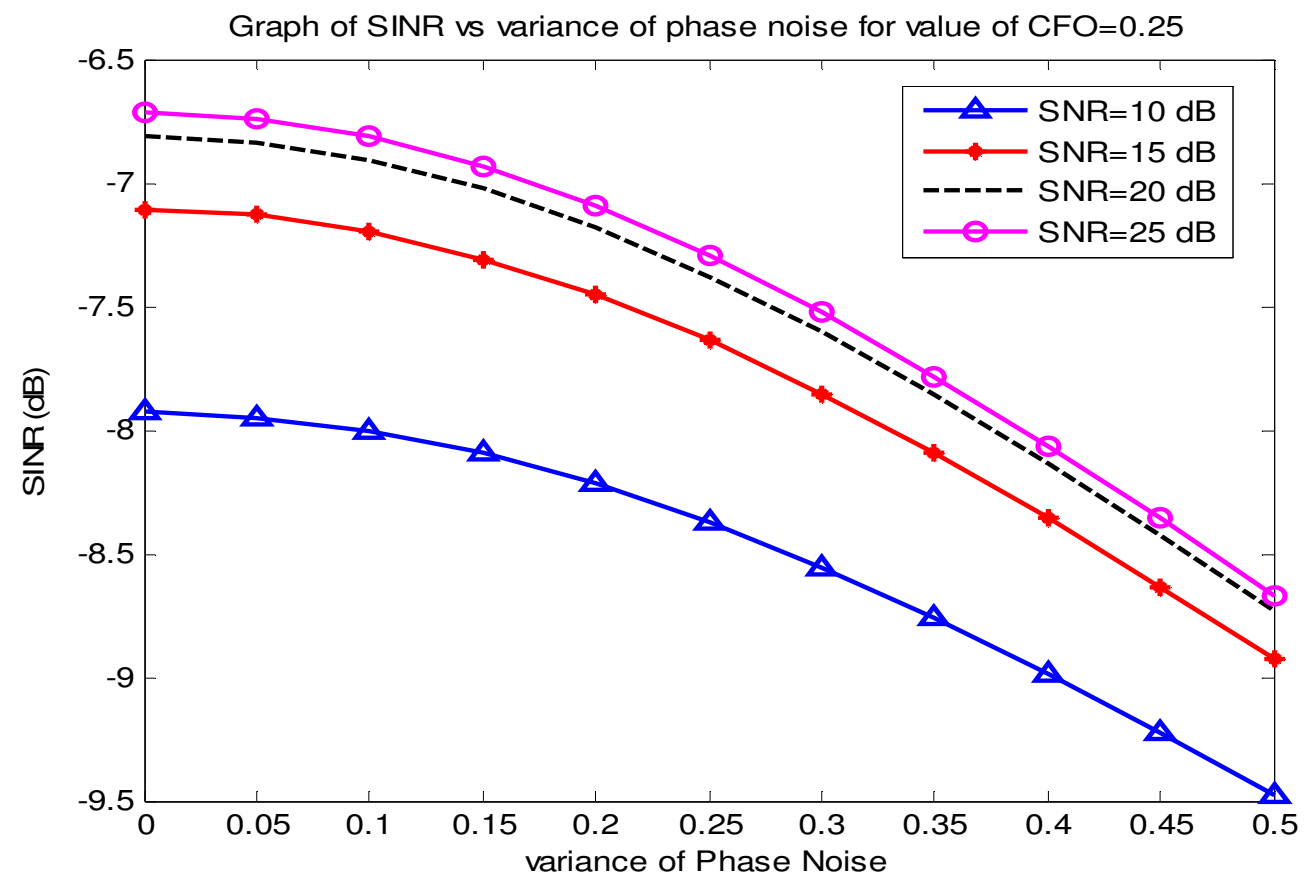

Fig. 9 SINR versus variance of phase noise (normalized $\mathrm{CFO}=0.25$ )

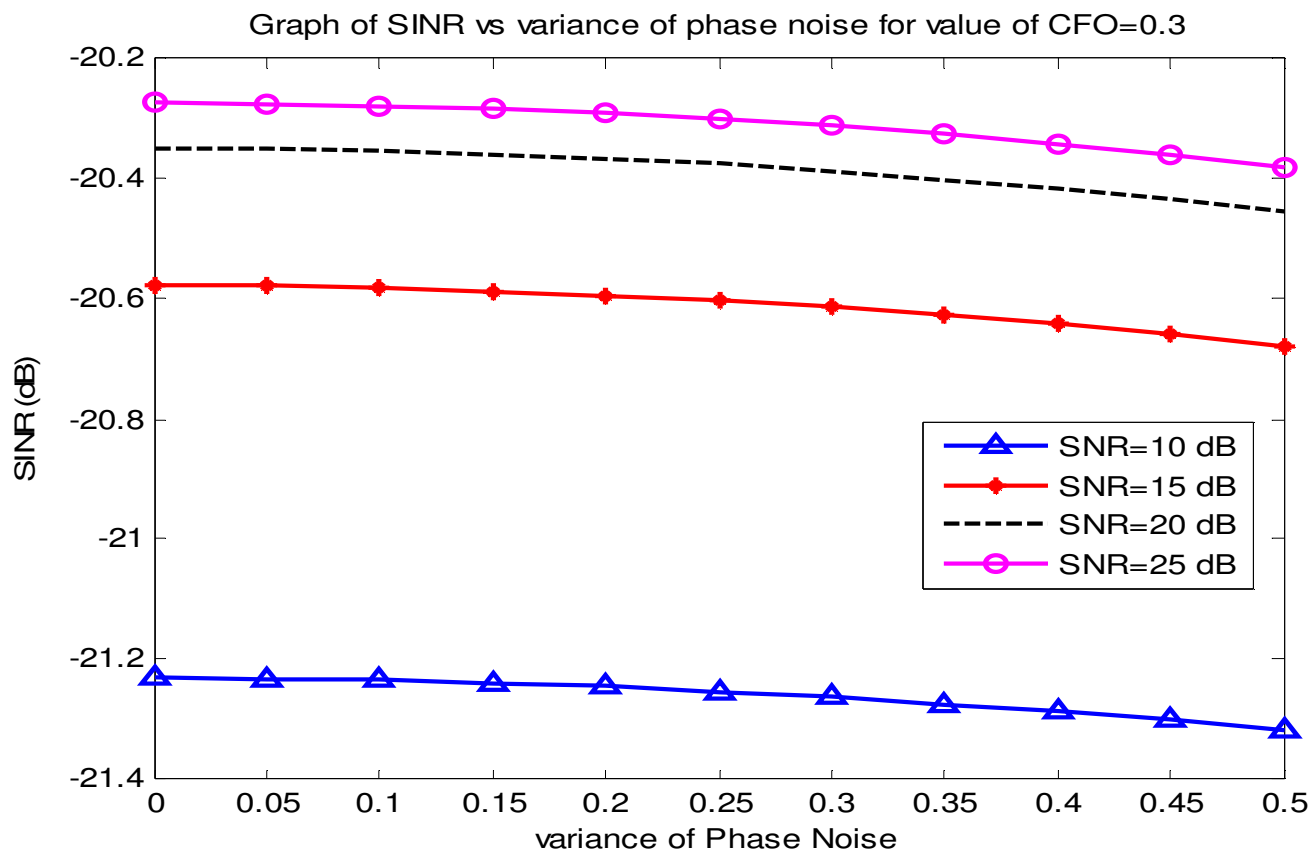

Fig. 10 SINR versus variance of phase noise (normalized $\mathrm{CFO}=0.3$ )

Results show that for different input SNR and for a fixed value of one of the synchronization 
errors (normalized CFO/variance of phase noise), SINR of the system undergoes penalty as the other error is increased.

\section{Conclusions}

In this paper, performance evaluation of an OFDM communication system is done by taking normalized CFO and variance of phase noise under consideration over Rayleigh fading channel. Simulation results show that for a particular value of variance of phase noise, SINR reduces as value of normalized CFO increases. Similarly, the SINR is reduced as variance of phase noise is increased for fixed normalized CFO. Hence it can be seen that OFDM system undergoes severe SINR penalty under the influence of synchronization errors. It is shown by analysis that the system performance also depends on several critical parameters such as number of subcarriers, input SNR and the fading characteristics of the channel. Further investigation can be carried out in this field by taking other important parameters into consideration, which are responsible for degrading the performance of OFDM communication system.

\section{REFERENCES}

[1] IEEE PES HVDC \& FACTS Subcommittee meeting (2006), IEEE PES Technical Committee Meeting, Montreal.

[2] T. Pollet, M. van Bladel, and M. Moeneclaey, "BER sensitivity of OFDM systems to carrier frequency offset and Wiener phase noise," IEEE Transaction on Communication, vol. 43, no. 2/3/4, pp. 191-193, Feb./Mar./Apr. 1995.

[3] M. Moeneclaey, "The effect of synchronization errors on the performance of orthogonal frequency-division multiplexed (OFDM) systems," in Proc. COST 254 (Emergent Techniques for Communication Terminals), Toulouse, France, July 1997.

[4] L. Tomba, "On the effect of Wiener phase noise in OFDM systems," IEEE Transactions Communication, vol. 46, no. 5, pp. 580-583, May 1998.

[5] A. G. Armada and M. Calvo, "Phase noise and sub-carrier spacing effects on the performance of an OFDM communication system," IEEE Communication Letter, vol. 2, no. 1, pp. 11-13, Jan. 1998.

[6] Steendam and M. Moeneclaey, "Synchronization Sensitivity of Multicarrier Systems," Euro. Trans. Telecomms., vol. 15, pp. 223-234, 2004.

[7] M. Luise and R. Reggiannini, "Carrier frequency acquisition and tracking f or OFDM systems," IEEE Transactions Communication, vol. 44, pp. 1590-1598, Nov. 1996.

[8] B. Le Floch, M. Alard, and C. Berrou, "Coded orthogonal frequency division multiplex," Proc. IEEE, vol. 83, no. 6, pp. 982-996, June 1995.

[9] Shankhanaad Mallick, Satya Prasad Majumder, "Performance Analysis of an OFDM System in the Presence of Carrier Frequency Offset, Phase Noise and Timing Jitter over Rayleigh Fading Channels," 5th International Conference on Electrical and Computer Engineering ICECE 2008, 20-22 December 2008, Dhaka, Bangladesh. 
International Journal of Distributed and Parallel Systems (IJDPS) Vol.3, No.3, May 2012

[10] Ana Garcia Armada, "Understanding the effects of phase noise in orthogonal frequency division multiplexing (OFDM)," IEEE transactions on broadcasting, vol. 47, no. 2, June 2001.

[11] Yu Zhang, Huaping Lui, "MIMO-OFDM system in the presence of Phase Noise and Doubly selective fading”, IEEE Transactions on Vehicular Technology, Vol. 56, No. 4, July 2007.

[12] Defeng Huang, Khaled Ben Letaif, "Carrier Frequency Offset Estimation for OFDM Systems using Null Subcarriers", IEEE transaction on Communications, Vol. 54, No. 5, May 2006.

[13] Mathecken, P. Riihonen, T. Werner, S. Wichman, "Performance Analysis of OFDM with Wiener Phase Noise and Frequency Selective Fading Channel," IEEE Trans. Communication, vol. 59, Issue 5, pp. 1321-1331, May 2011.

\section{Authors}

Gurpreet kaur was born in India on February 25, 1987. She received her B.Tech degree in Electronics and Communication from University Institute of Engineering and Technology, Kanpur University, India in 2009, and is currently in M. Tech second year(Signal Processing), Mody Institute of Technology and science (Deemed University), Rajasthan, India. She is currently working on OFDM technology.

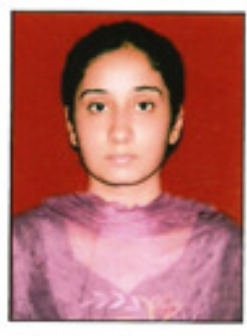

Partha Pratim Bhattacharya was born in India on January 3, 1971. He received M. Sc in Electronic Science from Calcutta University, India in 1994, M. Tech from Burdwan University, India in 1997 and Ph.D (Engg) from Jadavpur University, India in 2007.

He has 15 years of experience in teaching and research. At present he is working as Professor in Department of Electronics and Communication Engineering in the Faculty of Engineering and Technology, Mody Institute of Technology and Science (Deemed University), Rajasthan, India. He has published 60 papers in refereed journals and conferences. His broad research

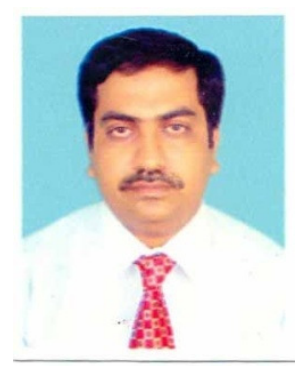
interest includes wireless communication.

Dr. Bhattacharya is a member of The Institution of Electronics and Telecommunication Engineers, India and The Institution of Engineers, India. He received Young Scientist Award from International Union of Radio Science in 2005. He is working as the editorial board member and reviewer in many reputed journals. 